\title{
Scimitar syndrome: A new multipatch technique and incidence of postoperative pulmonary vein obstruction
}

Robert L. Geggel, MD, ${ }^{\mathrm{a}, \mathrm{b}}$ Kimberlee Gauvreau, ScD, ${ }^{\mathrm{a}, \mathrm{b}}$ Ryan Callahan, MD, ${ }^{\mathrm{a}, \mathrm{b}}$ Eric N. Feins, MD, ${ }^{\mathrm{c}, \mathrm{d}}$ and Christopher W. Baird, MD ${ }^{\mathrm{c}, \mathrm{d}}$

\section{ABSTRACT}

Objective: A review of our center's experience before March 2011 showed that one half of 36 patients who had a baffling or reimplantation procedure to repair scimitar syndrome developed pulmonary vein obstruction. We analyzed the results of a new operation that enlarges the left atrium and avoids circuitous pathways or tension on the scimitar pulmonary vein.

Methods: Between April 2011 and November 2018, 22 patients underwent scimitar vein surgery; 11 had baffling or reimplantation and 11 only had the new operation that included resection of the atrial septum with removal of the muscular limbus. The left atrium was pulled down toward the scimitar vein and a V-shaped incision made at the scimitar vein atrial junction with the space filled with a pulmonary homograft. If the scimitar vein coursed adjacent to the atrium, a V-shaped incision was made into the scimitar vein and directly anastomosed to the atrium. A patch of autologous pericardium was used to septate the atrium and an additional patch placed anteriorly to augment the inferior vena cava.

Results: Of the 11 patients who had baffling or reimplantation, 5 developed pulmonary vein obstruction between 45 days and 9.5 months after surgery associated with baffle thrombosis or tension on the pulmonary vein. None of the 11 patients who only had the new procedure developed pulmonary vein obstruction during postoperative monitoring up to 3.6 years.

Conclusions: Patients having only the multipatch procedure for repair of scimitar syndrome have not developed postoperative pulmonary vein obstruction in the short to intermediate term. (JTCVS Techniques 2020;4:208-16)

From the Departments of a Cardiology, and ${ }^{\mathrm{c}}$ Cardiac Surgery, Boston Children's Hospital; and ${ }^{\mathrm{b}}$ Departments of Pediatrics and ${ }^{\mathrm{d}}$ Surgery, Harvard Medical School, Boston, Mass.

Accepted for the 100th Annual Meeting of The American Association for Thoracic Surgery.

Received for publication June 26, 2020; revisions received June 26, 2020; accepted for publication July 28, 2020; available ahead of print Aug 1, 2020.

Address for reprints: Robert L. Geggel, MD, Department of Cardiology, Boston Children's Hospital, 300 Longwood Ave, Boston, MA 02115 (E-mail: robert.geggel@ cardio.chboston.org).

2666-2507

Copyright (c) 2020 The Authors. Published by Elsevier Inc. on behalf of The American Association for Thoracic Surgery. This is an open access article under the CC BY-NC-

ND license (http://creativecommons.org/licenses/by-nc-nd/4.0/).

https://doi.org/10.1016/j.xjtc.2020.07.027

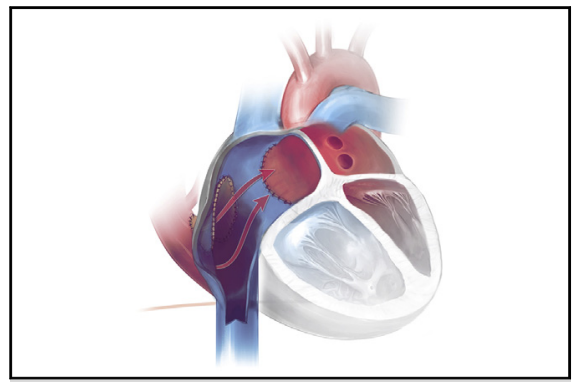

New surgical technique enlarges left atrium and avoids kinking or tension on pathway.

CENTRAL MESSAGE

A new multipatch technique is described for repair of scimitar syndrome that in the short to intermediate term has not been associated with postoperative pulmonary vein obstruction.

\section{PERSPECTIVE}

Surgical repair of scimitar syndrome using reimplantation or standard baffles is often associated with postoperative pulmonary vein obstruction. We describe a multipatch technique that enlarges the left atrium, brings it closer to the scimitar vein, avoids baffle kinking or tension on the anastomosis of the pulmonary vein to the atrium, and enables unobstructed flow in the short to intermediate term.

See Commentaries on pages 217, 219, and 221.

Scimitar syndrome is a form of partial anomalous pulmonary venous connection consisting of drainage of the right pulmonary veins to the inferior vena cava (IVC). The anomalously draining pulmonary veins often are evident on a chest radiograph as a curvilinear structure that resembles a sword, a pattern that provides the basis for the condition's name. ${ }^{1}$ Patients with scimitar syndrome have variable degrees of right lung hypoplasia and dextrocardia, and they 


\section{Abbreviations and Acronyms \\ $\mathrm{CMR}=$ cardiac magnetic resonance \\ $\mathrm{IVC}=$ inferior vena cava}

may have aortopulmonary collateral arteries to the hypoplastic lung or other structural congenital cardiac defects.

A variety of surgical procedures have been reported to redirect the anomalous pulmonary veins to the left atrium. $^{2-11}$ A previous review of our medical center's surgical experience from 1970 to March 2011 identified 36 patients who had scimitar vein surgery, one half of whom developed postoperative pulmonary vein obstruction (Figure 1) with no significant difference in rate of this complication regarding procedure (reimplantation or baffling) and with a trend toward increased occurrence when surgery was performed in infancy. ${ }^{12}$ The purpose of this report is to review results of a new operative technique that avoids circuitous pathways inherent in baffle procedures or tension on pulmonary venous connections.

\section{METHODS \\ Patients}

All patients with the diagnosis of scimitar syndrome who had cardiac surgery involving redirection of the anomalous pulmonary vein from the IVC to the left atrium performed at our medical center from April 2011 to November 2018 were identified from a search of a computerized database. Informed consent for surgery was obtained for all patients (institutional review board number and approval date: P00024172 10/11/2016). The study was approved by the Committee on Clinical Investigation at the medical center. Medical records were reviewed retrospectively.

\section{Surgical Technique}

Baffling and reimplantation techniques have been reported for surgical treatment of scimitar veins. These procedures involve placement of a baffle from the orifice of the scimitar vein near the IVC to an existing or surgically created atrial septal defect with potential additional baffling of the IVC (Figure 1) or excision of the scimitar vein from the IVC and reimplantation to either the left atrium or right atrium with baffling through an atrial septal defect. $^{11}$

The novel surgical technique incorporated a double- or triple-patch repair depending on the distance between the scimitar vein and atrial tissue (Figures 2 and 3). Autologous pericardium was harvested, fixed for $2 \mathrm{mi}-$ nutes in $0.6 \%$ glutaraldehyde, and rinsed. The scimitar vein was dissected free. Cardiopulmonary bypass was established, cardioplegia administered, and diastolic arrest achieved. If the scimitar vein was near the right atrialIVC junction, an IVC cannula was placed inferiorly in a typical position; if the scimitar vein entered at or below the diaphragm, an open-drop suction catheter was used. A right atriotomy was performed. The atrial septum was fully resected critically removing the area of the muscular limbus (Figure 2, $B$ and $C$ ). This resection included the back wall of the left atrium to that the heart was exited posteriorly. The back wall of the left atrium was pulled rightward and downward toward the IVC to shorten the distance from the scimitar vein (Figure 2, $C$, insert) and run together with interlocking polypropylene suture. A right lateral atriotomy in the posterior part of the right atrium was made and opened down at a right angle into the scimitar vein creating a V-shaped incision (Figure 2,D). This space was patch augmented with a thin pulmonary homograft with running polypropylene

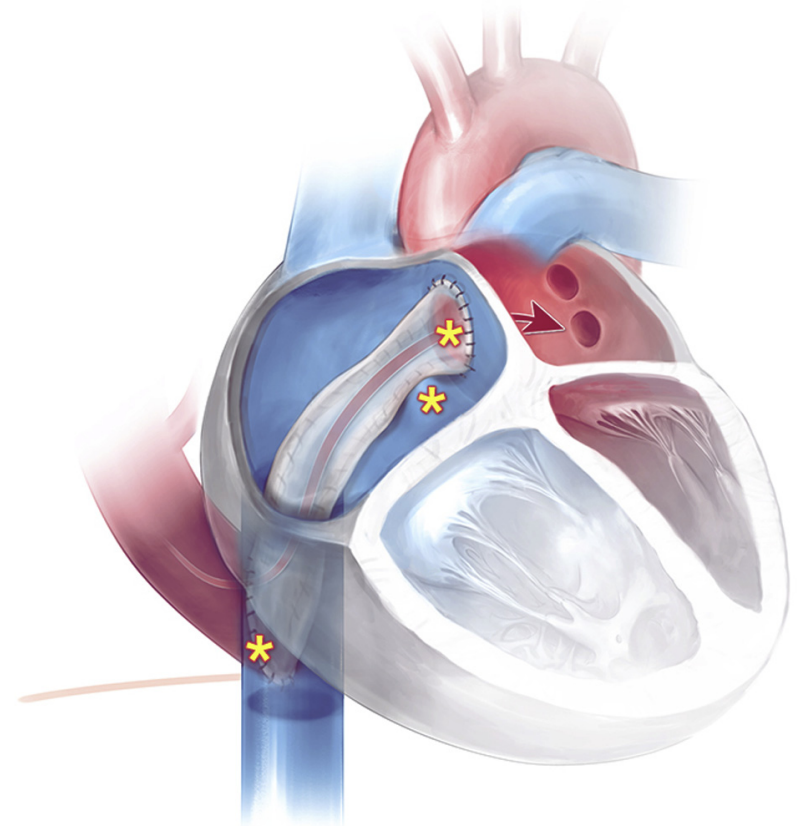

FIGURE 1. Sites of obstruction associated with traditional baffle repair of scimitar syndrome. Diagram depicts traditional baffle repair of scimitar syndrome. The stars depict sites of potential obstruction associated with the long angulated course of the scimitar vein baffle pathway, including at the proximal segment of the scimitar vein baffle pathway, superior limbic band, and atrial septal defect.

suture in an L-shaped fashion effectively enlarging the left atrium, taking any tension or torsion off the scimitar vein so as not to pull it too tightly to the left atrium, and shortening the scimitar pulmonary venous pathway. A pulmonary homograft patch was chosen rather than a pericardial patch since it is more likely to conform to angles and not kink. If the scimitar vein coursed adjacent to the left or right atrium, a V-shaped incision was made in the vein and the atrium was pulled down into this region and directly connected to the scimitar vein with running polypropylene suture without kinking or tension. The scimitar vein was not detached from the IVC to not change the angulation of the scimitar vein. This avoids acute angle changes in the scimitar vein where it meets the lung parenchyma, where a fulcrum effect could lead to obstruction. Atrial septation was accomplished with a second patch of autologous pericardium that was sewn between the IVC and patched scimitar vein and carried up onto the superior limbus with running polypropylene suture completely closing the atrial septal defect and separating the IVC from the left atrium (Figure 2, E). The IVC was opened anteriorly down to the diaphragm and a third patch of autologous pericardium with running polypropylene suture was used to augment the IVC area where the atrial septation baffle patch had been placed (Figure 2, F). The right atriotomy was closed, heart deaired, aortic crossclamp removed, patient warmed, and weaned from cardiopulmonary bypass.

\section{Anticoagulation}

Patients were started on heparin in the immediate postoperative period and then on aspirin, tested for sensitivity before discharge, and maintained for 3 months.

\section{Hemodynamic Data}

Pulmonary-to-systemic flow ratio was assessed by cardiac magnetic resonance (CMR) or cardiac catheterization. ${ }^{13}$ Pulmonary pressure 

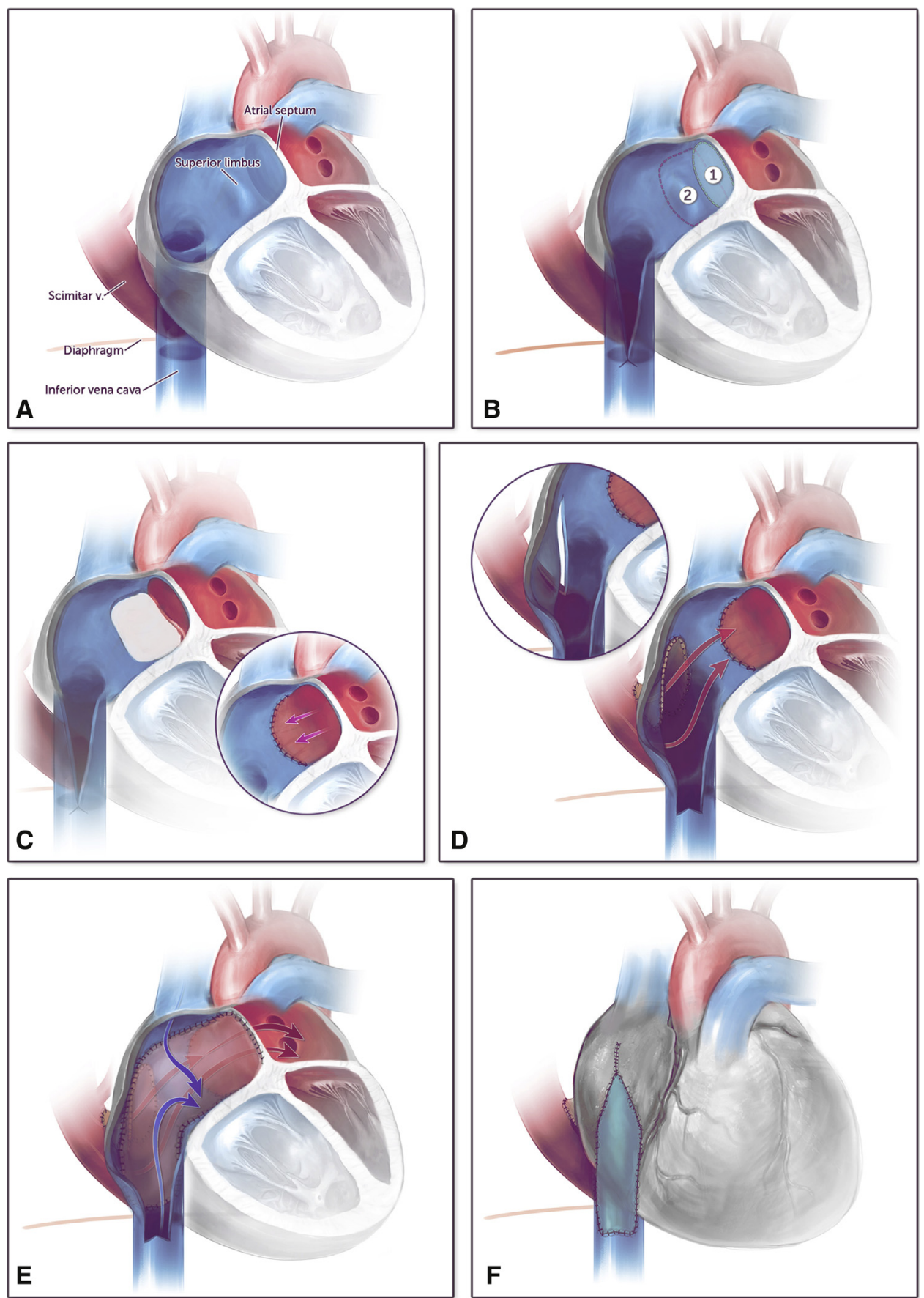

FIGURE 2. Triple patch operative technique to repair scimitar syndrome. A, Baseline anatomy showing scimitar vein connecting to the IVC superior to the level of the diaphragm and intact atrial septum. B, An incision is made in the right atrium and IVC to identify the origin of the scimitar vein. The atrial septum (1) and superior limbic band (2) are imaged. C, The atrial septum and superior limbic band have been resected. The left atrium is pulled downward and anastomosed to the right atrial tissue bringing the left atrium closer to the scimitar vein. D, A V-shaped incision is made in the atrium and scimitar vein and the space is filled in with a thin pulmonary homograft. E, An autologous pericardial patch is placed to divide the scimitar vein from the IVC and direct flow from the scimitar vein via a relatively straight baffle to the left atrium (red arrows) effectively closing the atrial septal defect. The vena cava flow courses anterior to this patch (blue arrows). F, A pericardial patch is placed in the IVC in the region where the baffle patch was placed to "raise the roof" and augment the caliber of this region.

(greater or less than one-half systemic level) was assessed by cardiac catheterization or echocardiography using the tricuspid valve regurgitation jet velocity or position of the interventricular septum.
Differential pulmonary blood flow was assessed by CMR or radionuclide lung perfusion scans. Ventricular volumes were assessed by CMR. 

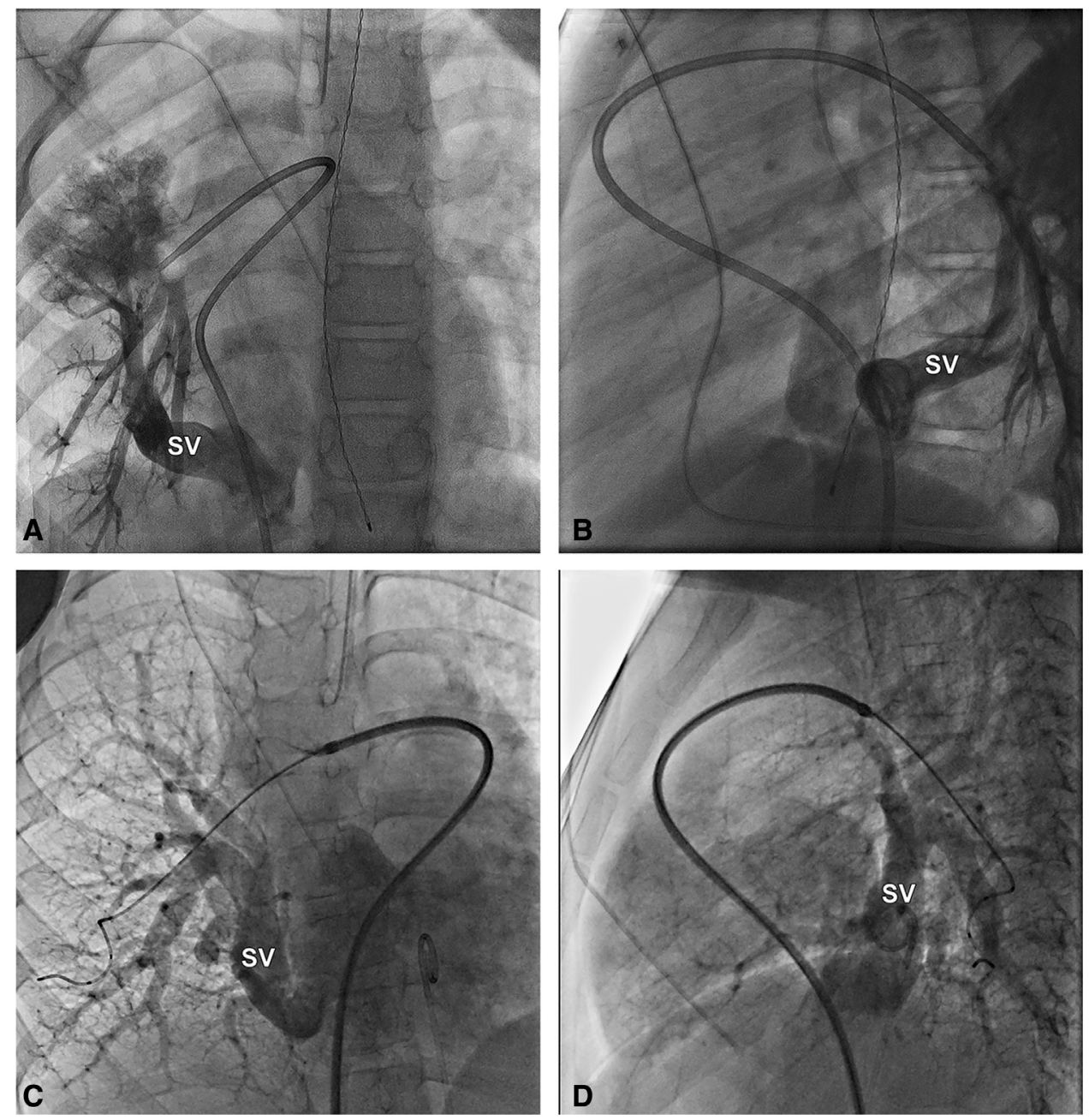

FIGURE 3. Preoperative cineangiograms. Preoperative anteroposterior and lateral views of levophase of right pulmonary artery angiogram in a 7-year-old patient who had the SV coursing a distance from the atrium and who subsequently had the triple multipatch procedure using pulmonary homograft insertion to connect the SV to the atrial tissue (A, B) and in a 10-year-old patient who had the SV in close proximity to the atrium and who subsequently had direct connection of the SV to the atrial tissue without use of pulmonary homograft material (C, D). SV, Scimitar vein.

\section{Definition of Pulmonary Vein Obstruction}

Pulmonary vein stenosis was identified by Doppler echocardiography by nonphasic flow or spectral Doppler velocity $>1.6 \mathrm{~m} / \mathrm{s}$ in the pulmonary vein, or discrete stenosis detected by computed tomography. The stenosis was confirmed at cardiac catheterization by direct injection of contrast into individual pulmonary veins on angiography.

\section{Data Analysis}

Patient characteristics were summarized using median (range) for continuous variables and number (percent) for categorical variables. Comparisons between the 2 surgical groups were performed using the Wilcoxon rank sum test for continuous variables and Fisher exact test for categorical variables. Time from repair to postoperative stenosis was estimated using the Kaplan-Meier method, and compared between groups using the logrank test.

\section{RESULTS}

\section{Patient Characteristics}

Twenty-two patients had surgery to redirect the scimitar vein at the IVC junction to the left atrium during the study period. Of these patients, 4 had a baffling procedure, 7 had reimplantation, and 11 only had the new multipatch procedure. Demographic and clinical features are presented in Table 1. There was no significant difference in the age at diagnosis or age at surgery in patients who had reimplantation or baffling compared with patients who had the multipatch technique. The median postoperative echocardiography followup was shorter for patients who had the multipatch procedure but with one exception was longer than the latest detection of obstruction in the baffling or reimplantation group, and the difference did not reach statistical significance. Of the patients who had the multipatch procedure, 1 patient referred from another nation had limited echocardiography followup of 1 month; the other 10 patients had echocardiography performed 10 months to 3.6 years after surgery. Aortopulmonary collateral arteries were identified in 12 patients and were occluded during catheterization performed before surgical repair in 11. One patient had a small collateral that was not 
TABLE 1. Patient characteristics

\begin{tabular}{|c|c|c|c|}
\hline Variable & $\begin{array}{c}\text { Multipatch procedure } \\
(\mathbf{n}=\mathbf{1 1})\end{array}$ & $\begin{array}{l}\text { Baffle or reimplantation } \\
\qquad(\mathbf{n}=\mathbf{1 1})\end{array}$ & $P$ value \\
\hline Age at diagnosis, $y$ & $0.2(1 \mathrm{~d}, 9.5)$ & $0.5(1 \mathrm{~d}, 8.3)$ & .79 \\
\hline Age at surgery, y & $3.7(5 \mathrm{~d}, 15.7)$ & $3.7(0.7,10.6)$ & .49 \\
\hline Imaging follow-up, y & $1.0(29 \mathrm{~d}, 3.6)$ & $2.2(0.6,4.2)$ & .20 \\
\hline Sex male & $7(64 \%)$ & $6(55 \%)$ & 1.0 \\
\hline APC & $6(55 \%)$ & $6(55 \%)$ & 1.0 \\
\hline Extracardiac anomalies & $6(55 \%)^{*}$ & $4(36 \%) \dagger$ & .67 \\
\hline \multicolumn{4}{|l|}{ Cardiac anomalies excluding ASD } \\
\hline VSD and coarctation & 1 & & \\
\hline RUPV-azygos vein & 1 & & \\
\hline DORV & & 1 & \\
\hline LUPV-innominate vein & & 2 & \\
\hline LUPV-LSVC stenosis & & 1 & \\
\hline LLPV stenosis & & 1 & \\
\hline Holmes heart, DILV & & 1 & \\
\hline LSVC-coronary sinus & & 5 & \\
\hline \multicolumn{4}{|l|}{ Clinical presentation } \\
\hline Respiratory symptoms & 4 & 4 & \\
\hline Murmur evaluation & 2 & 1 & \\
\hline Dextrocardia on chest radiograph & 1 & 3 & \\
\hline Follow-up fetal echocardiogram & $3 \ddagger$ & $1 \ddagger$ & \\
\hline Incidental diagnosis & & $2 \S$ & \\
\hline Unknown & 1 & & \\
\hline
\end{tabular}

Values are given as median (range) or number. $A P C$, Aortopulmonary collateral; $A S D$, atrial septal defect; $V S D$, ventricular septal defect; $R U P V$, right upper pulmonary vein; $D O R V$, double-outlet right ventricle; $L U P V$, left upper pulmonary vein; $L S V C$, left superior vena cava; $L L P V$, left lower pulmonary vein; $D I L V$, double inlet left ventricle. *Vertebral anomaly, imperforate anus, laryngeal cleft, cataract, renal dysplasia. $†$ Tracheoesophageal fistula/esophageal atresia, imperforate anus, vertebral anomaly, thumb hypoplasia, hydrocephalus, tracheobronchomalacia. §Neonatal echocardiogram to follow-up on concerns raised from fetal study, including possible coarctation; coarctation and VSD; unable to identify right pulmonary veins; dextroposition. §Echocardiogram to evaluate previously diagnosed ASD; noted at catheterization in patient with double-inlet left ventricle.

occluded. No patient who had occlusion of an aortopulmonary collateral artery developed clinically apparent pulmonary infarction.

\section{Hemodynamics}

Hemodynamic data from cardiac catheterization, nuclear perfusion scans, and cardiac magnetic resonance imaging studies are summarized in Table 2. Four patients had the degree of left-to-right shunting assessed at baseline and after coil occlusion of aortopulmonary collateral arteries with the ratio of pulmonary to systemic flow decreasing from 2.8 to $1.6,4$ to $1.5,2.2$ to 1.8 , and 2.4 to 1.5 , respectively. Systolic pulmonary pressure greater than one-half systemic level was present in 4 of 15 patients diagnosed in the first year of life and in 0 of 7 patients diagnosed later $(P=.26)$. Scimitar vein surgery was performed in the first year of life only in the 4 patients with pulmonary hypertension, one of whom had the multipatch operation at 5 days of age and had follow-up for 3.6 years and 3 of whom had reimplantation at 7 to 8 months of age $(P=.59)$. The degree of right lung hypoplasia as assessed by percent flow to the right lung varied among the patients.

\section{Baffle Procedure}

Four patients had placement of a pericardial baffle to redirect the scimitar vein to the left atrium via a surgically created

TABLE 2. Hemodynamic parameters at presentation and postoperative scimitar vein obstruction

\begin{tabular}{lccc}
\hline \multicolumn{1}{c}{ Variable } & Multipatch procedure & Baffle or reimplantation & $\boldsymbol{P}$ value \\
\hline Qp/Qs $(\mathrm{n}=11,10)$ & $2.0(1.6-2.8)$ & $2.3(1.6,4.0)$ & .48 \\
Systolic PAP $>1 / 2$ systemic $(\mathrm{n}=11,11)$ & 1 & 3 & .59 \\
RVEDVi, $\mathrm{mL} / \mathrm{m}^{2}(\mathrm{n}=6,3)$ & $118(103-204)$ & $126(73-144)$ & .90 \\
Right lung perfusion, $\mathrm{n}(\%)(\mathrm{n}=6,10)$ & $42(28-55)$ & $39(21-52)$ & .74 \\
Postoperative SV obstruction $(\mathrm{n}=11,11)$ & 0 & $5(45 \%)$ & .017 \\
\hline
\end{tabular}

Values are given as total number or median with range. $Q p / Q s$, Ratio of pulmonary/systemic flow; $P A P$, pulmonary artery pressure; RVEDVi, right ventricular end-diastolic volume index; $S V$, scimitar vein. 
atrial septal defect $(n=2)$ or enlargement of a secundum atrial septal defect $(\mathrm{n}=2)$. In 1 patient, a back-wall connection between the left atrium and back wall of the scimitar pulmonary vein was performed to decrease the distance between the pulmonary vein and left atrium. A separate pericardial baffle was placed to augment the diameter of the IVC-right atrial junction in 3 patients. Two of these patients had coexisting anomalous drainage of the left upper pulmonary vein to the innominate vein repaired by anastomosing that vein to the left atrial appendage.

\section{Reimplantation Procedure}

Seven patients had division of the scimitar vein at its junction with the IVC and reimplantation in the atrium. In 1 patient with double-inlet left ventricle, the scimitar vein was connected to the atrium posterior to location of a baffle used to construct a lateral tunnel Fontan pathway; in this patient, the scimitar vein was spatulated at the anastomosis site. In 2 patients, the scimitar vein was divided from the IVC without a button of surrounding tissue, anastomosed to the right atrium, and a pericardial patch placed to redirect the vein through a surgically created atrial septal defect. One of these patients had previous surgical ligation of an aortopulmonary collateral artery to the right lower lobe at an outside hospital and developed severe stenosis of the inferior vena cava at the right atrial junction and mild stenosis of the scimitar vein in the region of the surgical clip. At catheterization performed before scimitar vein surgery, balloon dilation relieved both sites of obstruction. This patient also had sutureless repair of left pulmonary vein stenosis produced by compression of this vein where a persistent left superior vena cava crossed the left pulmonary vein-left atrial junction.

In 3 patients, the scimitar vein was directly anastomosed to the left atrium with access to the left atrium via an atrial communication (patent foramen ovale $[\mathrm{n}=2]$ or inferior sinus venosus atrial septal defect $[\mathrm{n}=1]$ ) that was enlarged. In 1 patient, the scimitar vein was divided at its insertion site in the IVC, spatulated and anastomosed to the left atrium. In another patient, the scimitar vein was cut out as a single button containing some atrial wall and had sufficient length to reach the left atrium without tension. In the third patient, the scimitar vein was removed from the IVC with a large cuff of tissue and reimplanted in the left atrium; the IVC defect was closed with autologous pericardium and a separate pericardial patch was used to reconstruct the atrial septum.

In 1 patient, a hybrid procedure was performed. In this patient, the scimitar vein entered low in the IVC, was divided and anastomosed to the right atrium, and its flow directed to the left atrium using the triple multipatch procedure. This patient had double-outlet right ventricle and stenosis of both the scimitar vein at the IVC junction and the left upper pulmonary vein, where it connected anomalously with a persistent left superior vena cava; both sites of obstruction were balloon dilated at the referring medical center before

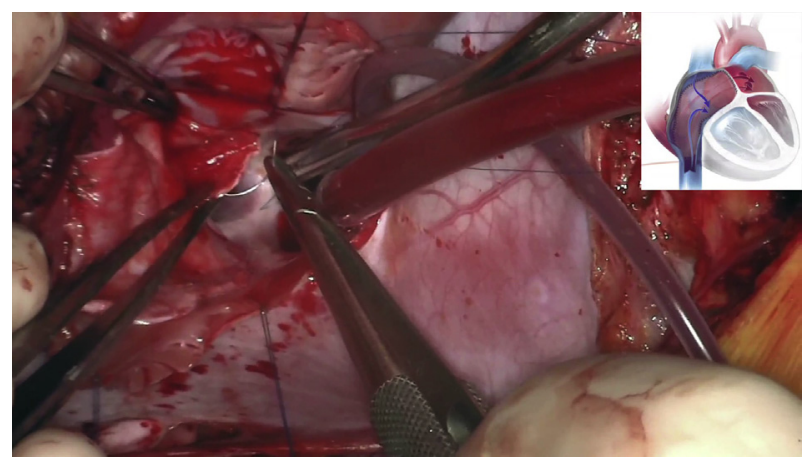

VIDEO 1. Before establishing cardiopulmonary bypass, the scimitar vein and inferior vena cava are widely mobilized off the pericardial reflection laterally and inferiorly along the diaphragm. Cardiopulmonary bypass is established and the right atrium is opened. The right atriotomy is extended superiorly and then inferiorly. The intracardiac anatomy is explored demonstrating the scimitar vein laterally. The interatrial septum is incised entering the left atrium. The atrial septal tissue is then extensively resected including the superior limbus and the muscular portion of the interatrial septum. This resection includes the back wall of the left atrium thus exiting the heart posteriorly. The back wall of the left atrium is brought rightward and laterally toward the scimitar vein and sewn in place with a running 6-0 polypropylene suture, which effectively shortens the distance between the scimitar vein and the left atrium. The lateral portion of the back wall of the right atrium is incised. This incision is extended inferiorly into the scimitar vein. A patch of pulmonary homograft is fashioned and sewn into the back wall of the left atrium and pulmonary venous pathway to fill in the gap created by the incision. A patch of autologous pericardium is cut to be used to close the atrial septal defect and baffle the scimitar vein to the left atrium. The suture line starts at the junction of the inferior vena cava and the scimitar vein and is carried superiorly and laterally along the ridge of the scimitar vein. The patch is large enough so there is a patulous pathway between the scimitar vein and left atrium. The superior aspect of the patch is closed. The inferior vena cava cannula is then removed and pump suckers are placed in the inferior vena cava to control systemic venous return. The inferior vena cava is incised inferiorly and a patch of autologous pericardium is placed to augment the inferior vena cava to right atrium pathway. The patch is carried up laterally and superiorly along the right atrial wall. After the patch is sewn in place, the right atriotomy is closed primarily with 2 layers of running 5-0 polypropylene suture. Video available at: https://www.jtcvs.org/article/S2666-2507(20)30365-5/fulltext.

surgery with no residual obstruction. This patient also had the left upper pulmonary vein reimplanted into the left atrial appendage, reimplantation of the left superior vena cava to the right atrium, and fenestrated patch closure of the ventricular septal defect. This patient was categorized in the reimplantation group because it was felt the reimplantation changed the angulation of the scimitar vein and was the predisposing factor for obstruction.

\section{Multipatch Procedure}

Six patients had the scimitar vein coursing a distance from right atrium and required the triple patch technique (Figure 3, $A$ and $B$, and Video 1). One patient had the right upper pulmonary vein connecting to the azygos vein repaired by clipping the azygos vein, placement of a 

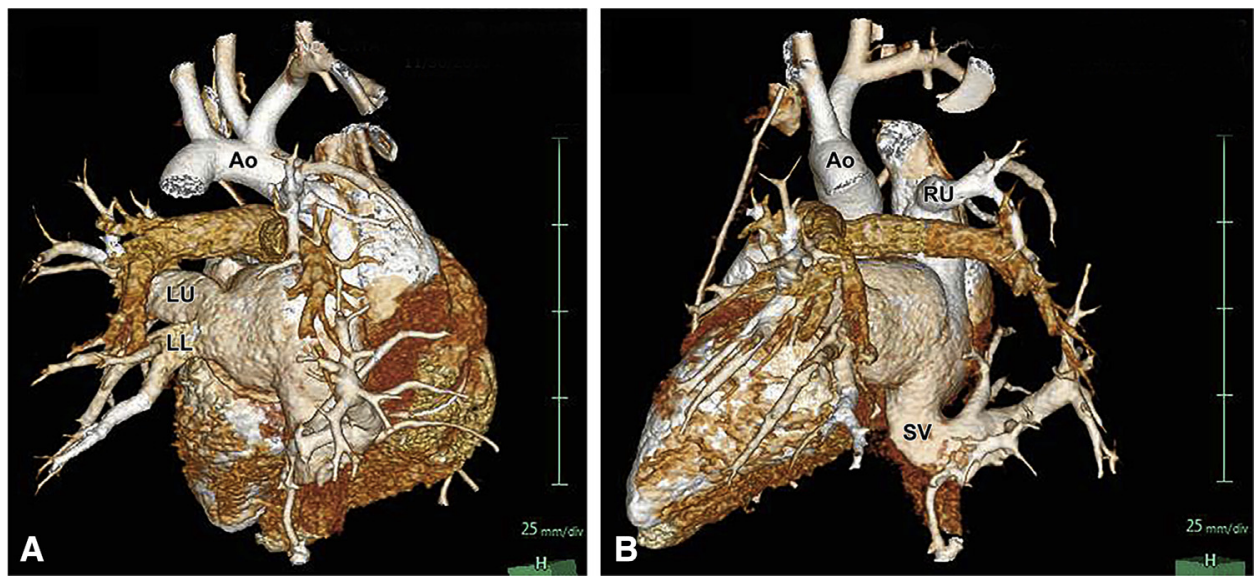

FIGURE 4. Postoperative cardiac computed tomography scan. Postoperative computed tomography scan of 7-year-old child who had the triple multipatch procedure as part of surgical repair. Unobstructed left upper $(L U)$ and left lower $(L L)$ pulmonary veins connecting to the left atrium are shown in (A) and unobstructed scimitar vein $(S V)$ is shown in (B) This patient also had the right upper $(R U)$ pulmonary vein connecting to the azygos vein and had this vein baffled separately to the left atrium. Ao, Aorta.

pericardial patch to direct the pulmonary venous flow to the left atrium, and a separate pericardial patch to augment the superior vena cava-right atrial junction. Five patients had the scimitar vein coursing close to the atrium and had the double patch technique. One of these patients also had patch closure of a muscular ventricular septal defect and coarctectomy with arch repair (Figure 3, $C$ and $D$ ).

\section{Postoperative Pulmonary Vein Stenosis}

None of the 11 patients who only had the multipatch technique developed scimitar vein obstruction during the follow-up period (Figures 4 and 5). Five of the 11 patients who had baffling $(\mathrm{n}=2)$ or reimplantation ( $\mathrm{n}=3$ including the patient with a hybrid procedure) developed obstruction in the scimitar vein documented 45, 46, 84, 215, and 284 days after surgery $(P=.017$ comparing this group with the multipatch group) (Figure 5). The 2 patients who were diagnosed with obstruction more than 3 months after surgery lived out of state and were diagnosed at initial postoperative clinic visits at our medical center. No patient was diagnosed with scimitar pulmonary vein stenosis later than 9.5 months after surgery. Stenosis developed in 2 of 4 patients who had surgery $<1$ year of age and in 3 of 18 who had surgery later $(P=.21)$. In 1 patient who had a baffle

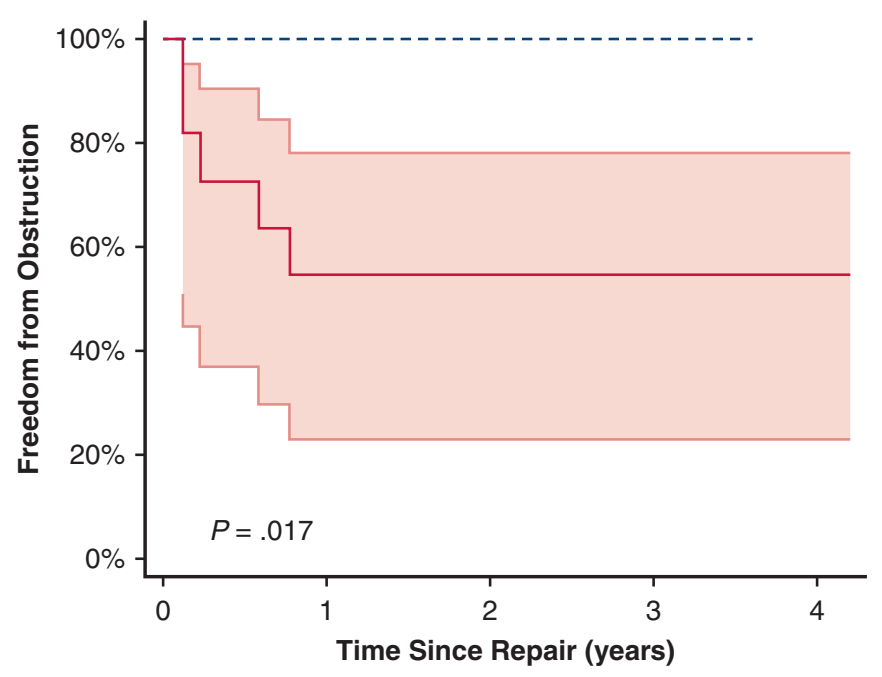

Number at risk

$\begin{array}{llllll}-- \text { New Procedure } & 11 & 9 & 4 & 1 & 0 \\ \text { - Baffle or Reimplantation } & 11 & 5 & 4 & 4 & 3\end{array}$

FIGURE 5. Freedom from scimitar vein obstruction after surgical repair. Kaplan-Meier curve depicting freedom from development of scimitar vein obstruction after standard repair (baffling or reimplantation) and the new procedure using double or triple patches as described in the text. The variable length of follow-up is depicted by the number of patients at risk over the study period. The shaded area depicts the confidence band for the baffle or reimplantation group. Confidence bands could not be constructed for the new procedure group, since no patients developed obstruction. 
there was in situ thrombosis at the junction of the scimitar vein with the baffle; this region was balloon dilated with no evidence for recurrent obstruction as assessed by echocardiography 1 year later. In the other patient with a baffle, a back-wall connection between the left atrium and the back wall of the scimitar pulmonary vein performed to decrease the distance between the pulmonary vein and left atrium created tension on the scimitar vein that produced longsegment stenosis that was not amenable to treatment. All 3 patients who developed obstruction after reimplantation had the scimitar vein separated from the IVC without a cuff of IVC or right atrial tissue. One of these patients had the pulmonary vein spatulated before reimplantation lateral to a Fontan baffle and developed stenosis at the anastomotic site possibly due to increased tension on the vein. The 2 other patients with reimplantation were younger than 1 year of age at the time of surgery. Each had preoperative scimitar vein obstruction, one associated with a surgically placed clip to occlude an aortopulmonary collateral and the other congenital obstruction, both of which were balloon dilated with no obstruction before surgery. This last patient had a hybrid procedure and developed scimitar vein obstruction that extended proximally that was judged to be associated with the pulmonary vein anastomosis rather than the baffle patch. These 3 patients had the obstruction treated with a variety of balloon dilation, stent placement and surgical patch or sutureless repair.

\section{Postoperative Arrhythmia}

One 7-year-old patient 3 weeks after a triple multipatch procedure developed postpericardiotomy syndrome associated with fever, chest pain, and pleural and pericardial effusions. At this time, he had nonsustained episodes of ectopic atrial tachycardia at rate 270 beats per minute controlled with esmolol infusion and then transitioned to oral sotalol. The patient was last evaluated 2 months after surgery and was in sinus rhythm. The patient returned home to another nation, and no additional follow-up information was available. No patient has required placement of a pacemaker.

\section{DISCUSSION}

Similar to our earlier report, ${ }^{12}$ nearly one half the patients who had reimplantation or baffling developed postoperative obstruction of the scimitar vein. The acute angulation between the baffle and the scimitar vein as well as tension on a reimplanted vein can be contributing factors. Obtaining a margin of atrial or IVC tissue when dividing the scimitar vein from the IVC may contribute to a more patulous subsequent reimplantation of the pulmonary vein to an atrium $^{11}$; both patients who had such an anastomosis did not develop obstruction, whereas 3 of 5 patients who had direct reimplantation of scimitar vein tissue did. The obstruction occurred early after surgery, often in the first
3 months, with detection identified less than 9.5 months after surgery in patients seen later for initial follow-up. Pulmonary artery systolic pressure greater than 0.5 systemic level was present only in patients diagnosed in the first year of life, although this did not reach statistical significance. There were no deaths in the current study, including 4 patients with pulmonary hypertension and 2 patients with preoperative left pulmonary vein obstruction, risk factors noted in our earlier report. ${ }^{12}$

In contrast, none of the patients who only had the multipatch operation developed postoperative scimitar vein obstruction during the study period, including one who had surgery in the first year of life. One patient who had a "hybrid" operation including both reimplantation and the multipatch technique did develop scimitar vein obstruction judged to be associated with the reimplantation. With the exception of 1 patient from another nation who had follow-up imaging 1 month after surgery, the other patients had echocardiography performed more than 9.5 months after the surgical procedure (10 months to 3.6 years), the time period during which obstruction was identified in the baffling or reimplantation group. This new procedure enlarges the left atrium and brings the left atrium closer to the scimitar vein to avoid kinking or tension on the anastomosis of the scimitar vein with the atrium thereby allowing the scimitar vein to flow more directly to the left atrium. The systemic venous pathway is augmented with a separate patch. The proximity of the scimitar vein to the atrium determines if an intervening thin pulmonary homograft patch is required to connect the scimitar vein to the atrial tissue.

There are roles for baffling or reimplantation techniques in selected patients. For example, adult patients may have room for placement of a generous baffle without kinking of the pathway whereas patients with a scimitar vein that is not restricted at its parenchymal entry and has adequate mobilization may have reimplantation without obstruction. Advantages of reimplantation may include not routinely requiring cardiopulmonary bypass and completion via a right thoracotomy. ${ }^{11}$

Multiple suture lines and incisions in the atria have been implicated in the occurrence of atrial arrhythmias in patients after Mustard or Senning atrial baffle procedures or lateral tunnel Fontan procedures. ${ }^{14,15}$ The multipatch procedure does involve extensive suturing at the atrial level. Postoperative arrhythmia was identified after the multipatch procedure in 1 patient who developed ectopic atrial tachycardia early after repair that coincided with the occurrence of postpericardiotomy syndrome, which may have been contributory. Longer-term follow-up is necessary to assess whether arrhythmias will develop in this patient group.

Not all patients with scimitar syndrome need surgical intervention. Long-term complications of an atrial level shunt can include atrial arrhythmias from chronic right atrial dilation, right ventricular dysfunction from chronic 
right ventricular volume overload, and pulmonary hypertension from increased pulmonary flow. ${ }^{16}$ There is variable right lung hypoplasia with scimitar syndrome, and some patients may have dual drainage of the right pulmonary veins to both the IVC and left atrium so that the degree of left-toright shunting may not cause right-sided chamber dilation or pulmonary hypertension. ${ }^{10}$ In patients with scimitar syndrome, aortopulmonary collateral arteries can also contribute to elevated pulmonary pressure..$^{8,12,17,18}$ If present, initially closing the collateral arteries is beneficial with subsequent reassessment of hemodynamics prior to deciding on the need for surgery. Occluding the collateral arteries does not cause pulmonary infarction, since the lung tissue is supplied by pulmonary and bronchial arterial circulation. ${ }^{2,8,10,17,19}$ In the absence of right-sided chamber enlargement, pulmonary hypertension, or other structural congenital heart disease requiring surgery, observation is reasonable. If indicated, surgical repair using the multipatch technique can provide acceptable results regardless of age. The degree of left-to-right shunting can increase with age due to changes in relative left and right atrial pressures so that serial monitoring is required to ensure that a clinically significant shunt does not develop. ${ }^{20}$

\section{Study Limitations}

This study has several limitations, including single institution, retrospective review, and small sample size. Importantly, there was only one neonate in the multipatch group, and although this patient did not have postoperative obstruction, more neonatal patients need to show a similar outcome to be confident about this finding. In addition, while postoperative pulmonary vein obstruction usually occurs within the first few months after surgery, and the shortto intermediate-term results are encouraging, additional follow-up will be useful to ensure that no long-term complications associated with the novel procedure develop. The majority of the new multipatch procedures were performed by the senior surgeon, and familiarity with the procedure is required before the expected outcomes may be seen.

\section{CONCLUSIONS}

Patients having only the multipatch procedure for repair of scimitar syndrome have not developed postoperative pulmonary vein obstruction in the short to intermediate term. This procedure avoids both circuitous pathways associated with standard baffle placement as well as tension on the scimitar vein associated with reimplantation. Additional follow-up will be useful to ensure that no long-term complications associated with the multipatch procedure develop.

\section{Conflict of Interest Statement}

Dr Feins reported CryoLife (consultant). All other authors reported no conflicts of interest.
The Journal policy requires editors and reviewers to disclose conflicts of interest and to decline handling or reviewing manuscripts for which they may have a conflict of interest. The editors and reviewers of this article have no conflicts of interest.

We thank Kai-ou Tang for preparation of Figures 1 and 2.

\section{References}

1. Neill CA, Ferencz C, Sabiston DC, Sheldon H. The familial occurrence of hypoplastic right lung with systemic arterial supply and venous drainage "scimitar syndrome." Bull Johns Hopkins Hosp. 1960;107:1-15.

2. Najm HK, Williams WG, Coles JG, Rebeyka IM, Freedom RM. Scimitar syndrome: twenty years' experience and results of repair. J Thorac Cardiovasc Surg. 1996;112:1161-9.

3. Canter CE, Martin TC, Spray TL, Weldon CS, Strauss AW. Scimitar syndrome in childhood. Am J Cardiol. 1986;58:652-4.

4. Dupuis C, Charaf LAC, Brevière GM, Abou P. "Infantile" form of the scimitar syndrome with pulmonary hypertension. Am J Cardiol. 1993;71:1326-30.

5. Dupuis C, Charaf LAC, Brevière GM, Abou P, Rémy-Jardin M, Helmius G. The "adult" form of the scimitar syndrome. Am J Cardiol. 1992;70:502-7.

6. Honey M. Anomalous pulmonary venous drainage of the right lung to the inferior vena cava ("scimitar syndrome"): clinical spectrum in older patients and role of surgery. Q J Med. 1977;46:463-83.

7. Huddleston CB, Exil V, Canter CE, Mendeloff EN. Scimitar syndrome presenting in infancy. Ann Thorac Surg. 1999;67:154-60.

8. Gao Y, Burrows PE, Benson LN, Rabinovitch M, Freedom RM. Scimitar syndrome in infancy. J Am Coll Cardiol. 1993;22:873-82.

9. Vida VL, Padalino MA, Boccuzzo G, Tarja E, Berggren H, Carrel T, et al. Scimitar syndrome- European Congenital Heart Surgeons Association (ECHSA) multicentric study. Circulation. 2010;122:1159-66.

10. Brown JW, Ruzmetov M, Minnich DJ, Vijay P, Edwards CA, Uhlig PN, et al. Surgical management of scimitar syndrome: an alternative approach. J Thorac Cardiovasc Surg. 2003;125:238-45.

11. Gudjonsson U, Brown JW. Scimitar syndrome. Semin Thorac Cardiovasc Surg Pediatr Card Surg Ann. 2006;9:56-62.

12. Dusenbery SM, Geva T, Seale A, Valente AM, Zhou J, Sena L, et al. Outcome predictors and implications for management of scimitar syndrome. Am Heart J. 2013;165:770-7.

13. Powell AJ, Tsai-Goodman B, Prakash A, Greil GF, Geva T. Comparison between phase-velocity cine magnetic resonance imaging and invasive oximetry for quantification of atrial shunts. Am J Cardiol. 2003;91:1523-5.

14. Rychik J, Atz AM, Celermajer DS, Deal BJ, Gatzoulis MA, Gewillig MH, et al. Evaluation and management of the child and adult with Fontan circulation: a scientific statement from the American Heart Association. Circulation. 2019;140:e234-84.

15. Butto F, Dunnigan A, Overholt ED, Benditt DG, Benson DW Jr. Transesophageal study of recurrent atrial tachycardia after atrial baffle procedures for complete transposition of the great arteries. Am J Cardiol. 1986;57:1356-62.

16. Geggel RL, Mark EJ. Case records of the Massachusetts General Hospital, case 37-1993. A 48 year-old woman with an atrial septal defect and pulmonary hypertension. N Engl J Med. 1993;329:864-72.

17. Uthaman B, Abushaban L, Al-Qbandi M, Rathinasamy J. The impact of interruption of anomalous arterial supply on scimitar syndrome presenting during infancy. Catheter Cardiovasc Interv. 2008;71:671-8.

18. Dickinson DF, Galloway RW, Massey R, Sankey R, Arnold R. Scimitar syndrome in infancy- role of embolization of systemic arterial supply to right lung. Br Heart J. 1982;47:468-72.

19. Haworth SG, Sauer U, Bühlmeyer K. Pulmonary hypertension in scimitar syndrome in infancy. Br Heart J. 1983;50:182-9.

20. Alpert JS, Dexter L, Vieweg WVR, Haynes FW, Dalen JE. Anomalous pulmonary venous return with intact septum-diagnosis and pathophysiology. Circulation. 1977;56:870-5.

Key Words: congenital heart disease, scimitar syndrome, partial anomalous pulmonary venous return, pulmonary vein stenosis 